\title{
A Fuzzy Control-Based Service Configuration Approach for Ubiquitous Computing Applications
}

\author{
Yong ZHANG ${ }^{\dagger a)}$, Student Member, Shensheng ZHANG ${ }^{\dagger}$, Nonmember, and Songqiao $\mathrm{HAN}^{\dagger}$, Student Member
}

SUMMARY This paper proposes a novel service configuration approach that can realize dynamic critical Quality of Service (QoS) adaptation to ever-changing and resource-limited ubiquitous computing environments. In the approach, service configuration is reduced to a Fuzzy Control System (FCS) which aims to achieve critical QoS variations on minimal level with less power cost. Two configuration strategies, service chain reconfiguration and QoS parameters adjustment, along with a configuration algorithm, are implemented to handle different types of QoS variations. A self-optimizing algorithm is designed to enhance the adaptation of the FCS. Simulation results validate the proposed approach.

key words: service configuration, fuzzy control, ubiquitous computing, quality of service

\section{Introduction}

Recently, Ubiquitous Computing (UbiComp) has attracted many researchers' interest [1] and various UbiComp applications, such as 3D tourism navigation application TellMaris and pervasive retail system MyGrocer [2], have been developed based on mobile devices and wireless networks. But in ever-changing UbiComp environments, the applications, especially multimedia applications, still encounter two main problems: 1) environmental variations introduce instable network connections and bandwidth which could cause the serious fluctuations of critical QoS; 2) resource limitations make it difficult for the applications to meet critical QoS requirements of end users persistently. To solve the above problems, we need to develop an adaptive service configuration approach that can realize dynamic critical QoS adaptation with effective resource utilization. The term "service configuration" in this paper refers to the methodology of guaranteeing the applications' QoS, which includes resource allocation, service composition and service component adjustment.

In fact, service configuration for distributed applications and mobile applications has been widely studied. Ferrari et al. proposed reserving network connections to ensure QoS [3]. Li et al. presented a hybrid PID-fuzzy control method to allocate CPU and bandwidth resource for QoS adaptation [4]. But these approaches involving resource reservation and reallocation are infeasible for resourceconstrained UbiComp applications. Xu et al. employed the

\footnotetext{
Manuscript received September 1, 2008.

Manuscript revised December 27, 2008.

${ }^{\dagger}$ The authors are with the Department of Computer Science and Engineering, Shanghai Jiao Tong University, 200240 Shanghai, China.

a) E-mail: chengshan@ @jtu.edu.cn DOI: 10.1587/transinf.E92.D.1186
}

different compositions of service components to enable the application with the different levels of QoS [5]. But their service composition approach easily causes great $\mathrm{QoS}$ variations in changeful environments. Moreover, their work does not take account of resource cost.

To obtain satisfying critical QoS, we try to dynamically regulate the application's internal structure and parameters to adapt to the changing and resource-limited environments. An UbiComp application can be considered as a directed task graph $G=\{V, E\}$ across mobile-end and server-end which are connected by GPRS, Bluetooth, $3 \mathrm{G}$ and $\mathrm{WiFi}$, etc [6]. Where, $V$ is a set of vertexes which specify the requested service components to accomplish the tasks, and $E$ is a set of edges that represent dependency relationships between the tasks. Figure 1 shows a simplified task graph of a wireless video monitor application. The task $u_{0}$ can capture images by the built-in camera of a mobile phone, $u_{1}$ can transcode images by altering the color depth of images, $u_{2}$ and $u_{5}$ are responsible for images encryption and decryption, $u_{3}$ and $u_{4}$ are used to send and receive data over wireless network, $u_{6}$ can display the images. Each task may be executed by a number of alternative service components. In runtime, an instantiation of the application can be modelled as a configurable service chain which has an impact on critical QoS. Moreover, each service component may contain tunable QoS parameters that can also affect critical QoS. Hence, we adopt service component-level and service chain-level configuration to improve critical QoS with less resource cost. For simplicity, this paper only considers the power cost of mobile devices, and focuses on the applications with one critical QoS attribute and represented by directed acyclic task graph.

This paper proposes a novel service configuration approach for UbiComp applications. Dynamic service configuration is reduced to a FCS which can handle non-linear configuration process. Two configuration strategies are designed and implemented to support adaptive critical QoS control. Based on simulated annealing, a self-optimizing algorithm for the key parameters of the FCS is designed to

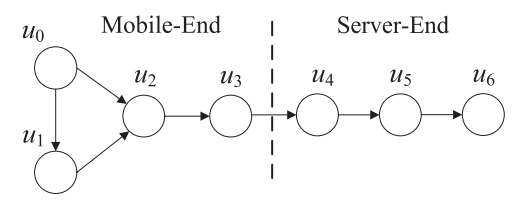

Fig. 1 Task graph of wireless video monitor application. 
enforce the adaptation of the approach.

\section{Our Approach}

In this section, we first present service configuration model and the architecture of the FCS, then detail the two configuration strategies, a configuration algorithm and the selfoptimizing algorithm. Finally, we conduct some simulations to validate the proposed approach.

\subsection{Fuzzy Control System for Service Configuration}

Usually, an application $G$ may have multiple candidate service chains denoted as $G_{c}=\left\{c_{1}, c_{2}, \ldots, c_{m}\right\}$. We use $S\left(c_{k}\right)$ to represent the set of the service components in service chain $c_{k}(k \in[1, m])$. The power cost of a service chain $c_{k}$ equals the total power cost of all service components in $S\left(c_{k}\right)$ :

$$
R_{c}\left(c_{k}\right)=\sum_{s_{i} \in S\left(c_{k}\right), c_{k} \in G_{c}} R_{s}\left(s_{i}\right)
$$

Where, $R_{s}\left(s_{i}\right)$ represents the power cost of $s_{i}$ which can be obtained through power profiling method [7].

Service configuration for UbiComp applications can be considered as a dynamic critical QoS control process. Due to power constraint and environmental variations, the control process is complicated and non-linear. Thus, we apply fuzzy control method, a well-known solution to non-linear systems, to deal with such a control process. Besides, the rule-based fuzzy inference can be flexibly updated and expanded to improve control performance. In our approach, we use Average Absolute Deviation (AAD) to measure QoS variations, and use $R_{G}$ to denote the power cost of the application $G$. In view of inevitable critical QoS variations and limited power, service configuration is reduced to a FCS which aims to achieve minimal AAD of critical QoS with less $R_{G}$.

$$
\begin{aligned}
& \mathrm{AAD}=\left(\left(\int_{0}^{T_{G}}|e(t)| d t\right) / T_{G}\right) \\
& R_{G}=\sum\left(T_{s}\left(s_{i}\right) \cdot R_{s}\left(s_{i}\right)\right)
\end{aligned}
$$

Where, $e(t)$ denotes critical QoS error which is the deviation of actual critical QoS from the desired value. $T_{G}$ is the duration of configuration process. $T_{s}\left(s_{i}\right)$ denotes the total execution time of service component $s_{i}$ in the configuration process.

Figure 2 illustrates the main components of the FCS and the whole service configuration process. Firstly, context manager periodically collects and delivers context information associated with actual critical QoS and power cost as the feedback in the configuration process. Service configurator serves as a controller that manipulates the output of configuration instructions depending on power cost, critical QoS error and error change, etc. Finally, the concrete configuration operations are conducted by service adapters under the configuration instructions. We also design an optimization mechanism for key parameters of the FCS relying on a performance index, which is detailed in Sect. 2.4.

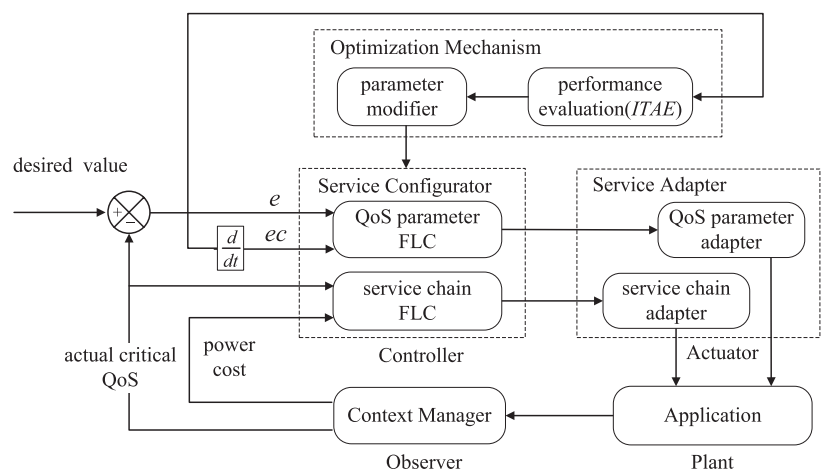

Fig. 2 Fuzzy control system for service configuration.

\subsection{Service Configuration Strategies}

As stated previously, critical QoS is influenced by the status of service chains and the service components in service chains. In our approach, two configuration strategies are designed and implemented with two Fuzzy Logic Controllers (FLCs) in the service configurator. Accordingly, the service configurator can produce two types of configuration instructions which are conducted by the service adapters as shown in Fig. 2.

(1) Service Chain Reconfiguration (SCR)

The status of service chains can influence the application's critical QoS greatly. Hence, we can alter the application's critical QoS by reconfiguring service chain, such as adding, deleting as well as substituting service components. In applying this strategy, the FLC inputs actual critical QoS and power cost, and then outputs service chain configuration instructions. The fuzzy rules can be described as follows:

$$
\text { rule }^{(i)}: \text { If } \tilde{V}_{1} \text { is } \tilde{\eta}_{1}^{i} \wedge \tilde{V}_{2} \text { is } \tilde{\eta}_{2}^{i} \ldots \wedge \tilde{V}_{m} \text { is } \tilde{\eta}_{m}^{i} \text { Then } \tilde{y} \text { is } \tilde{B}^{i}
$$

Where, $\tilde{V}_{j}(j=1,2, \ldots, m)$ are the input linguistic variables of critical QoS and power cost, and $\tilde{y}$ is the output linguistic variables of configuration instructions; $\tilde{\eta}_{j}^{i}(i=1,2, \ldots, n)$ and $\tilde{B}^{i}$ are the $i^{\text {th }}$ linguistic value of linguistic variable $\tilde{V}_{j}$ and $\tilde{y}$ respectively, which are characterized by membership functions $\mu_{\tilde{\eta}_{j}^{i}}\left(\tilde{V}_{j}\right)$ and $\mu_{\tilde{B}^{i}}(\tilde{y})$.

Each rule represents a fuzzy implication from critical QoS and power cost to a service chain configuration instruction: $\tilde{\eta}_{1}^{i} \times \tilde{\eta}_{2}^{i}, \ldots, \times \tilde{\eta}_{m}^{i} \rightarrow \tilde{B}^{i}$. If the $i^{t h}$ rule is fired by the FLC, the corresponding configuration instruction $\tilde{B}^{i}$ will be conducted by the service chain adapter to transform current chain $c_{k}$ into a new chain $c_{k}^{\prime}$. This process can be formalized as follows:

$$
c_{k}^{\prime}=\psi_{c_{k}}\left(\tilde{B}^{i}\right)
$$

Where, $\psi_{c_{k}}$ is a mapping function. To get an optimal configuration with less power cost, firstly a $\alpha$-cut set of configuration instructions is generated as a set of candidate instructions: $\tilde{B}_{\alpha}=\left\{\tilde{B}^{i}: \mu_{\tilde{B}^{i}}(\tilde{y}) \geq \alpha_{r}\right\}$. Where, $0<\alpha_{r} \leq$ 
$\max _{\tilde{B}^{i} \in \tilde{B}}\left\{\mu_{\tilde{B}^{i}}(\tilde{y})\right\}$ that guarantees $\tilde{B}_{\alpha} \neq \emptyset$. Then, a set of can$\tilde{B}^{i} \in \tilde{B}_{o}$ didate service chains can be obtained: $C_{\alpha}=\left\{c_{k}^{\prime}: c_{k}^{\prime}=\right.$ $\left.\psi_{c_{k}}\left(\tilde{B}^{i}\right), \tilde{B}^{i} \in \tilde{B}_{\alpha}\right\}$. Finally, according to Eq. (1) and Eq. (5), the optimal configuration instruction can be calculated as follows:

$$
\begin{aligned}
\tilde{B}^{*} \in \tilde{B}_{\alpha}^{*} & =\left\{\arg \min _{c_{k}^{\prime} \in C_{\alpha}} R_{c}\left(c_{k}^{\prime}\right)\right\} \\
& =\left\{\arg \min _{\tilde{B}^{i} \in \tilde{B}_{\alpha}} R_{c}\left(\psi_{c_{k}}\left(\tilde{B}^{i}\right)\right)\right\}
\end{aligned}
$$

If $\left\|\tilde{B}_{\alpha}^{*}\right\|>1$, then $\tilde{B}^{*}=\arg \max _{\tilde{B}^{i} \in \tilde{B}_{\alpha}^{*}}\left\{\mu_{\tilde{B}^{i}}(\tilde{y})\right\}$. Here, $\arg \max _{x}\left\{\mu_{x}(\tilde{y})\right\}$ returns the configuration instruction $x$ that results in the maximum $\mu_{x}(\tilde{y})$.

(2) QoS Parameter Adjustment (QPA)

The application's critical QoS is also relevant to the QoS of individual service components in the service chain, which is decided by service components' QoS parameters. Hence, we can adjust the application's critical QoS by tuning the values of QoS parameters in involved service components. We implement this strategy by applying TakagiSugeno fuzzy control model by which the FLC takes the error and the error change of critical QoS, and produces configuration instructions associated with the QoS parameters' values. Suppose there are two tunable QoS parameters $p_{0}$ and $p_{1}$, the fuzzy numbers sets of critical QoS error and error change are $L_{e}=\left\{\xi_{0}, \xi_{1}, \ldots, \xi_{m}\right\}$ and $L_{e c}=$ $\left\{\zeta_{0}, \zeta_{1}, \ldots, \zeta_{n}\right\}$, then the $i^{t h}$ functional rules can be described as follows:

$$
\text { If } \tilde{F}_{e}=\xi_{i} \wedge \tilde{F}_{e c}=\zeta_{i} \text { Then }\left\{\begin{array}{l}
p_{0}=\delta_{i, 0} \cdot \tilde{F}_{e}+\omega_{i, 0} \cdot \tilde{F}_{e c}+\gamma_{i, 0} \\
p_{1}=\delta_{i, 1} \cdot \tilde{F}_{e}+\omega_{i, 1} \cdot \tilde{F}_{e c}+\gamma_{i, 1}
\end{array}\right.
$$

Where, $\tilde{F}_{e}$ and $\tilde{F}_{e c}$ represent the linguistic variables of critical QoS error and error change respectively. $\xi_{i} \in L_{e}$ and $\zeta_{i} \in L_{e c} . \delta_{i, 0}, \delta_{i, 1}, \omega_{i, 0}, \omega_{i, 1}, \gamma_{i, 0}$ and $\gamma_{i, 1}$ are the coefficients for the $i^{\text {th }}$ fuzzy rule. To improve system response, according to Eq. (7), a fuzzy control table can be generated in advance. Thus, with the table, $p_{0}$ and $p_{1}$ can be quickly obtained by using $\tilde{F}_{e}$ and $\tilde{F}_{e c}$ as indexes.

\subsection{Configuration Algorithm}

Actually, the SCR is a coarse-grained strategy by which critical QoS can be adjusted sharply to deal with great critical QoS variations, but it tends to cause negative effects such as overshoot and oscillation. On the other hand, the QPA is a fine-grained strategy for slight critical QoS variations, but it takes longer time to reach the desired value. Hence, we develop a configuration algorithm which applies the two strategies dynamically depending on current critical QoS error, and application-specific range parameters of critical QoS error $\lambda_{a}$ and $\lambda_{b}\left(0<\lambda_{a}<\lambda_{b}\right)$. If critical QoS error is in $\left[-\lambda_{a},+\lambda_{a}\right]$, then the service configurator applies the QPA, else if critical QoS error is in $\left[-\lambda_{b},-\lambda_{a}\right]$ or $\left[\lambda_{a}, \lambda_{b}\right]$, then applies the SCR. Otherwise, both strategies are applied.

\subsection{Self-Optimization of Fuzzy Control System}

In order to improve the adaptation of the FCS, we apply simulated annealing [8] to automatically optimize key parameters, which include $\alpha_{r}$ for the $\alpha$-cut set of configuration instructions, and the range parameters $\lambda_{a}$ and $\lambda_{b}$ for configuration strategy selection. Moreover, we introduce Integral Time Absolute Error (ITAE), a widely-used performance index, as the objective function calculated by the following discrete form:

$$
\operatorname{ITAE}=t \cdot|e(t)| \cdot \Delta T
$$

Where, $\Delta T$ denotes sampling period. The self-optimizing iteration schema can be summarized as follows:

Step 1: initialize the parameters with user-specified values: $W_{\text {opt }}=\left[\alpha_{r}^{0}, \lambda_{a}^{0}, \lambda_{b}^{0}\right]$. According to Eq. (8), calculate the value of the objective function and assign it to variable ITAE $_{\text {pre }}$;

Step 2: generate a group of new parameters $W^{\prime}=f\left(W_{\text {opt }}\right)=$ $\left[\alpha_{r}^{\prime}, \lambda_{a}^{\prime}, \lambda_{b}^{\prime}\right]$. Where, $f$ is a perturbation function [9]. Then, calculate a new value of objective function and assign it to variable ITAE $_{\text {new }}$;

Step 3: calculate the accepting probability of the new parameters:

$$
p=\left\{\begin{array}{cl}
1 & \mathrm{ITAE}_{\text {new }}<\mathrm{ITAE}_{\text {pre }} \\
e^{\left(\mathrm{ITAE}_{\text {pre }}-\mathrm{ITAE}_{\text {new }}\right) / \Delta T} & \mathrm{ITAE}_{\text {new }} \geq \mathrm{ITAE}_{\text {pre }}
\end{array}\right.
$$

If $p>\tau(\tau=\operatorname{Random}(0,1)$ represents a random threshold value with a uniform distribution), then $W_{o p t}$ is updated with $W^{\prime}$. Otherwise, the new parameters are discarded.

Step 4: ITAE $_{\text {pre }}$ is updated with ITAE $_{\text {new }}$. Repeat Step 2 to Step 4 until ITAE $\mathrm{ITw}_{\text {new }}<\varepsilon_{0}$. Here, $\varepsilon_{0}$ is a specific minimal value.

\subsection{Simulation Experiments}

We have performed several simulations on a prototype of the wireless video monitor application. The critical QoS is frame rate and the desired value is set to $16 \mathrm{fps}$. In the simulations, we apply three service configuration approaches, including First-Order Logic for Service Composition (FOLSC) presented in [5], PID control for QoS Parameters Adjustment (PID-QPA) employed in [4], and our approach Fuzzy Control for Service Configuration (FC-SC), which are implemented with the assistance of JESS and fuzzy inference engine for Java [10]. Average Frame Rate (AFR), Average Absolute Deviation of Frame Rate (AADFR) and power cost are considered as performance metrics in the simulations. The sampling frequency is $50 \mathrm{~Hz}$. A $2 \mathrm{~KB}$, $160 * 120$ JPEG image with medium complexity is used as original images. We use a Nokia E61i mobile phone as a mobile-end, and two DELL 4600 PCs as a server-end and a router respectively. The router running NIST net can emulate various $3 \mathrm{G}$ wireless network characteristics, and the mobile-end is connected with the server-end by the router to emulate a $3 \mathrm{G}$ wireless network environment. In order to 
Table 1 Results of simulation experiments.

\begin{tabular}{|c|c|c|c|c|c|c|}
\hline \multirow{2}{*}{ Approach } & \multicolumn{2}{|c|}{ AFR (fps) } & \multicolumn{2}{c|}{ AADFR (fps) } & \multicolumn{2}{c|}{ PowerCost (mW) } \\
\cline { 2 - 7 } & SI & SII & SI & SII & SI & SII \\
\hline PID-QPA & 14.02 & 12.33 & 2.81 & 3.75 & 152 & 165 \\
\hline FOL-SC & 13.54 & 12.26 & 3.04 & 4.08 & 178 & 251 \\
\hline FC-SC & 15.75 & 14.87 & 2.57 & 3.11 & 132 & 163 \\
\hline
\end{tabular}

fully test our approach, we conduct the simulations in two scenarios: 1) Scenario I (SI): a user walks around an area with stable wireless network signals; 2) Scenario II (SII): a user enters and then leaves an area with unstable wireless network signals. The description of other experimental settings is omitted due to space limitation.

The simulation results are listed in Table 1. We find that in SI, the AFR with FC-SC reaches 15.75 fps which is higher than those with PID-QPA and with FOL-SC. And, the AADFR with FC-SC is $2.57 \mathrm{fps}$ that is less than those with PID-QPA and with FOL-SC. The results in SII show the similar characteristics. Particularly in SII, compared with PID-QPA and FOL-SC, FC-SC increases the AFR by $20.6 \%$ and $21.3 \%$ which are higher than $12.3 \%$ and $16.3 \%$ in SI, and FC-SC reduces the AADFR by $17.1 \%$ and $23.7 \%$ which are also higher than $8.5 \%$ and $15.5 \%$ in SI. These results show that our approach outperforms FOL-SC and PIDQPA both in AFR and AADFR, especially in the SII characterized with serious bandwidth fluctuation. As for power cost, FC-SC in SI consumes $132 \mathrm{~mW}$ which is $87 \%$ of PIDQPA and 74\% of FOL-SC. In SII, though the power cost of FC-SC is almost the same as that of PID-QPA, it is much less than that of FOL-SC by $35 \%$.

\section{Conclusion and Future Work}

In this paper, we propose an adaptive service configuration approach for mobile and resource-limited UbiComp applications. Self-optimizing fuzzy control method is applied to deal with the non-linear configuration process. Two configuration strategies are implemented to handle different types of QoS variations flexibly. Simulation results demonstrate that the proposed approach achieves better performances in improving critical QoS and utilizing limited power than other approaches.

This work needs to be studied deeply. How to optimize the membership functions and coefficients in the FLCs, and how they influence the approach performance need further exploration and evaluation. In addition, we plan to handle the potential rule explosion problem by applying hierarchical fuzzy inference technique. In another part of future work, we intend to extend the proposed approach for more complicated UbiComp applications represented by directed cyclic graph and with multiple critical QoS attributes and resource constraints.

\section{Acknowledgments}

This work is funded by grant 05SN07114 from the Shanghai Science and Technology Commission and the National Research Council of Canada.

\section{References}

[1] M. Satyanarayanan, "Pervasive computing: Vision and challenges," IEEE Pers. Commun., vol.8, pp.10-17, 2001.

[2] G. Roussos, A.J. Marsh, and S. Maglavera, "Enabling pervasive computing with smart phones," IEEE Pervasive Computing, vol.4, pp.20-27, 2005.

[3] D. Ferrari, A. Gupta, and G. Ventre, "Distributed advance reservation of real-time connections," Multimedia Syst., vol.5, pp.187-198, 1997.

[4] B. Li and K. Nahrstedt, "A control-based middleware framework for quality-of-service adaptations," IEEE J. Sel. Areas Commun., vol.17, pp.1632-1650, 1999.

[5] D. Xu, D. Wichadakul, and K. Nahrstedt, "Multimedia service configuration and reservation in heterogenous environments," Proc. IEEE International Conference on Distributed Computing Systems, pp.512-519, Taipei, 2000.

[6] S. Kalasapur, M. Kumar, and B.A. Shirazi, "Dynamic service composition in pervasive computing," IEEE Trans. Parallel Distrib. Syst., vol.18, no.7, pp.907-918, 2007.

[7] X. Feng, R. Ge, and K.W. Cameron, "Power and energy profiling of scientific applications on distributed systems," Proc. 19th IEEE International Parallel and Distributed Processing Symposium, pp.3443, Denver, CO, USA, 2005.

[8] E. Huyghe and Y. Hamam, "Simulated annealing for fuzzy controller optimization: Principles and applications," Proc. IEEE International Conference on Systems, Man and Cybernetics, pp.45094514, Vancouver, BC, Canada, 1995.

[9] P. Tian, J. Ma, and D.M. Zhang, "Application of the simulated annealing algorithm to the combinatorial optimisation problem with permutation property: An investigation of generation mechanism," Eur. J. Oper. Res., vol.118, pp.81-94, 1999.

[10] Clarkson University, Open Source Fuzzy Inference Engine for Java, http://people.clarkson.edu/ esazonov/FuzzyEn-gine.htm, 2000. 